\title{
Thrombozytenhemmung und -aktivierung
}

\author{
Mechanismen und klinische Implikationen
}

Lorenzo Alberio', Kenneth J. Clemetson²

\begin{abstract}
Zusammenfassung
Die Empfindlichkeit der Aktivierung von Thrombozyten ist Teil des delikaten Gleichgewichts, welches Hämostase von Thrombose unterscheidet. Unter physiologischen Bedingungen wird es durch die Hemmung der Thrombozytenaktivität und Entfernung von Agonisten erhalten. Unter pathologischen pro-
\end{abstract}

thrombotischen Bedingungen versucht der Mediziner, das Gleichgewicht mit Hilfe von Arzneimitteln wiederherzustellen. Die Resultate dieser Behandlungen verbessern sich stetig, aber dennoch sind ein besseres Verständnis der Mechanismen und die Suche nach alternativen Inhibitoren nach wie vor wichtig.

Schlüsselwörter: Thrombozyten · Aktivierungsmechanismen · Physiologie · Pathophysiologie $\cdot$ Thrombozytenhemmer

Herz 2005;30:176-80

DOI 10.1007/s00059-005-2681-x

\section{Platelet Inhibition and Activation. Mechanisms and Clinical Implications}

\begin{abstract}
The sensitivity to activation of platelets is part of the delicate equilibrium differentiating hemostasis from thrombosis. Under physiological conditions it is maintained by downregulating platelet activity and removing agonists. Under pathologic
\end{abstract}

conditions the clinician tries to restore this equilibrium with pharmaceutical drugs. The results obtained by such treatments are steadily improving but there is still need for better knowledge of the mechanisms involved and for alternative inhibitors.

Key Words: Platelets · Activation mechanisms · Physiology · Pathophysiology · Platelet inhibitors

\section{Einleitung}

Der Unterschied zwischen Hämostase, der normalen Fähigkeit, Blutverlust als Folge von Verletzungen zu verhindern, und Thrombose, der pathologischen Variante, bei der hauptsächlich die gleichen physiologischen Prozesse $\mathrm{zu}$ gewebeschädigenden Verschlüssen der Blutgefäße und zu Beschwerden wie Herzinfarkt und Schlaganfall führen, kann als Veränderung des Gleichgewichts der hemmenden und aktivierenden Prozesse, welche die Thrombozytenfunktion regulieren, gesehen werden. Natürlich sind nicht nur die Thrombozyten beteiligt, doch weil es komplexe Interaktionen zwischen Thrombozyten und Gefäßsystem (einschließlich Endo- thelium und Subendothelium) gibt, spiegelt sich ein Effekt, der auf eine der Komponenten wirkt, unweigerlich in anderen Komponenten wider.

\section{Physiologische Mechanismen, welche die} Thrombozyten in einem ruhenden Zustand halten Im normalen, gesunden Gefäßsystem wird die Thrombozytenaktivierung durch Endothelium und Subendothelium durch die Beeinflussung mehrerer kritischer Komponenten stark gesenkt.

1. Die plasmatische ADP-(Adenosindiphosphat-)Konzentration wird durch CD39, eine membrangebundene Nukleotidase, die auf der Endotheloberfläche liegt

\footnotetext{
Klinik und Poliklinik für Hämatologie und Hämatologisches

Zentrallabor der Universität, Inselspital, Bern, Schweiz,

${ }^{2}$ Theodor-Kocher-Institut, Universität Bern, Schweiz.
} 
und ADP und ATP (Adenosintriphosphat) rasch aus dem zirkulierenden Plasma abbaut, streng kontrolliert. Einerseits verhindert sie die Thrombozytenaktivierung durch beide Nukleotide, andererseits hält sie die Thrombozytenempfindlichkeit gegenüber diesen Agonisten durch Verhinderung des Herunterfahrens der Rezeptoren aufrecht. Die Hauptquellen dieser Nukleotide sind Erythrozyten und Thrombozyten, die bereits im normalen Blutkreislauf beschädigt oder aktiviert werden, weit mehr jedoch bei hohen Scherkräften infolge arterieller Verengungen, welche durch Arteriosklerose oder Bluthochdruck hervorgerufen werden.

2. Das Endothelium produziert $\mathrm{PGI}_{2}$ (Prostacyclin), das einerseits durch Regulierung der glatten Muskelzellen im Subendothelium die Gefäße erweitert hält und andererseits durch Hemmung der Signale zahlreicher Sieben-Transmembrandomäne-Rezeptoren der Thrombozyten (welche via G-Protein-Bahnen wie ADP-, Thrombin- und Thromboxanrezeptoren agieren) die Thrombozyten in einem ruhenden und diskoiden Zustand hält.

3. Das Endothelium produziert Stickoxid (NO), das ebenfalls auf die glatten Muskelzellen und die Thrombozyten einwirkt, um die Gefäße erweitert und die Thrombozyten ruhend zu halten. Dies geschieht durch Induktion der Bildung von zyklischem Guanosinmonophosphat (cGMP) und konsekutive Aktivierung cGMP-abhängiger Kinasen, die auch die G-Protein-Bahnen in analoger, aber nicht ganz identischer Weise als Prostacyclin beeinflussen.

4. Das Endothelium exprimiert neben Thrombomodulin Heparin und heparinähnliche Sulfatoligosaccharide enthaltende Glykosaminoglykanmoleküle, die Thrombin und Antithrombin binden können. Dies führt $\mathrm{zu}$ einer Inaktivierung jeglicher Spuren von Thrombin, welches im Kreislauf in einem nichthämostatischen Umfeld produziert wurde.

\section{Mechanismen der Thrombozytenaktivierung}

Jede Verletzung des Gefäßsystems, die zu einer Freilegung des Subendotheliums oder Aktivierung von Endothelzellen führt, bewirkt die Aktivierung ruhender Thrombozyten, um einen möglichen Blutverlust zu verhindern, eine nichtthrombotische Oberfläche neu zu formen und Reparaturprozesse anzuregen. Obwohl wir immer noch nicht alle Prozesse verstehen, die an der Bildung eines hämostatischen Pfropfs und dessen Regulation beteiligt sind, ist es möglich, wenigstens ein vereinfachtes Modell vorzuschlagen. Man beachte den einfachen Fall, bei dem eine oder mehrere Endothelzellen durch normale Abnutzung verloren gehen, ein Prozess, der in jeder Sekunde des Lebens abläuft und bei dem die Thrombozyten für den Unterhalt des Gefäßsystems eine entscheidende Rolle spielen. Für diese Funktion benötigen wir in der Regel ein Minimum von etwa 10000 Thrombozyten/ $\mu$ l Blut. Die Thrombozyten werden durch den Blutfluss gegen die Gefäßwände getrieben und interagieren mit ihnen, zuerst über den am exponierten subendothelialen Kollagen gebundenen Von-Willebrand-Faktor, welcher Glyokoprotein (GP) Ib von den Thrombozyten bindet. Es handelt sich um eine fließende Wechselwirkung, bei der Membranfäden, die von der Thrombozytenoberfläche gezogen werden, einen bremsenden Effekt auf den Thrombozyten ausüben, ihn verlangsamen und in einen intimeren Kontakt mit dem Subendothelium bringen.

Entweder befreit sich der Thrombozyt und zirkuliert weiter (sein zukünftiges Schicksal werden wir später erwägen), oder er wird durch GP Ib aktiviert, das andere Rezeptoren, speziell Integrine, ins Spiel bringt. Das erste beteiligte Integrin könnte $\alpha_{2} \beta_{1}$ sein, ein wichtiger Kollagenrezeptor; aber $\alpha_{\text {IIb }} \beta_{3}$ wird auch von GP Ib aktiviert und hat eine Bindungsstelle zum Von-Willebrand-Faktor. Der Kontakt zwischen Thrombozyten und Kollagen führt ebenso zu einer Wechselwirkung zwischen GP VI, dem anderen wichtigen Kollagenrezeptor, und subendothelialem Kollagen, welches eine stärkere Aktivierung der Thrombozyten verursacht. Dies umfasst die Aktivierung der Integrine $\alpha_{2} \beta_{1}$ und $\alpha_{\text {IIb }} \beta_{3}$, die Thrombozytenausbreitung und die Freisetzung von Granulainhalt. Der Prozess ist damit nicht beendet. Die aktivierten Thrombozyten, die das exponierte Subendothelium beschichten, bieten ihrerseits eine Angriffsfläche, an der sich andere Thrombozyten anhängen und aktiviert werden können. Hier wird kein Kollagen exponiert, und das weitere Anhaften und die Aktivierung geschehen durch andere anhaftende Proteine und Rezeptoren, insbesondere den Von-Willebrand-Faktor und Fibrinogen bzw. GP Ib und $\alpha_{\mathrm{IIb}} \beta_{3}$. Es folgen die Thrombozytenaktivierung und die Freisetzung von Granulainhalt. Dieser Prozess dauert an, bis das Gefäß entweder von einem wachsenden Thrombus verstopft ist, was u.U. zum Stoppen einer Blutung nötig ist, wenn das betroffene Gefäß durchschnitten wurde, oder - bei nur leichter Beschädigung der Gefäßwand bis die aktivierenden Effekte infolge der Stimulation via Subendothelium durch die beruhigenden Effekte 
der durch das umliegende Endothelium als Antwort auf die lokale Thrombinproduktion vermehrt sezernierten Faktoren ausgeglichen sind. Folglich wird das Thrombuswachstum irgendwann aufhören, und die unvollständig aktivierten äußeren Schichten des Thrombozytenthrombus verlieren ihre Fähigkeit, die vorbeiflieBenden Thrombozyten aufzufangen und zu aktivieren, sogar wenn sie mit ihnen in Kontakt kommen.

Die beschriebenen Vorgänge bilden nur ein Teil des Prozesses, da die Generation von Thrombin und die Stabilisierung des Thrombus durch Fibrin ebenfalls wichtige Komponenten der Hämostase sind. Durch Kollagen aktivierte Thrombozyten, speziell im Beisein von Thrombin, exprimieren mittels Durcheinanderwirbeln der Membranphospholipide eine prokoagulante Aktivität, indem sie damit negativ geladene Phospholipide an der Oberfläche freilegen. Dies erlaubt die Bildung von Prothrombinase und Xase-Komplexen und löst eine explosive Umwandlung von Prothrombin zu Thrombin aus. Das gebildete Thrombin wandelt Fibrinogen in Fibrin um; ebenso aktiviert es mehr Thrombozyten und auch angrenzende Endothelzellen. Das gebildete Fibrin hilft den Thrombus zu stabilisieren, und die Gerinnselretraktion (die durch Kontraktion der Thrombozyten-Aktomyosin zustande kommt, welches durch $\alpha_{\text {IIb }} \beta_{3}$ an Fibrin und somit an Nachbarthrombozyten gekoppelt ist) bewirkt, dass sich die Wundränder zusammenziehen.

\section{Labiles Gleichgewicht zwischen Hämostase und Thrombose}

Wenn sich die Thrombozyten in einem stärker aktivierten Grundzustand befinden oder leichter aktivieren lassen, dann verlagert sich das Gleichgewicht während der Thrombusbildung, und ein größerer Thrombus wird gebildet, bevor die ausgleichenden Antagonisten den Prozess stoppen und rückgängig machen können. In ähnlicher Weise bilden Thrombozyten in einem ruhigeren oder inhibierten Zustand unter gleichen Bedingungen einen kleineren Thrombus. Solche Unterschiede können bei pathologischen Bedingungen ausreichen, um den Verschluss der Blutgefäße mit allen damit einhergehenden Konsequenzen auszulösen oder zu verhindern. Man könnte auch sagen (obwohl das Konzept noch umstritten ist), dass der ruhende/aktivierte $\mathrm{Zu}$ stand der zirkulierenden Thrombozyten ein wichtiger Faktor bei der Entstehung von Arteriosklerose sein könnte [1]. Dies wird allerdings schwierig zu beweisen sein, da die Faktoren, welche die Reaktionsfähigkeit der Thrombozyten beeinflussen, auch auf die Endothelzellen und andere Komponenten des Gefäßsystems einwirken. Wie auch immer solche Wechselwirkungen hervorgerufen werden, sie werden rasch zu einem Teufelskreis, wo überaktive Thrombozyten die Gefäßwand beeinflussen bzw. ein überaktives Endothelium die Thrombozyten. Da diese Prozesse nicht linear, sondern exponentiell ablaufen, können kleine initiale Unterschiede letztendlich zu sehr großen Unterschieden führen.

Pathologische Zustände können entweder durch umweltbedingte oder genetische Faktoren entstehen. Die Tatsache jedoch, dass viele Kulturen, vor allem im Fernen Osten, seit Menschengedenken praktisch keine Herz-Kreislauf-Erkrankungen kannten und sich die Situation in neuerer Zeit rasch verschlechterte, zeigt an, dass umweltbedingte Faktoren eine vorherrschende Rolle spielen. Dazu gehören das Rauchen sowie Veränderungen in der Menge und Art des Fettkonsums, der Genuss von raffiniertem Zucker, die ungenügende $\mathrm{Zu}$ fuhr von Ballaststoffen, das Fehlen körperlicher Aktivität und der Stress. Während eine Umstellung einiger dieser negativen Faktoren helfen könnte, die epidemischen Herz-Kreislauf-Erkrankungen, welche die Welt (und im Speziellen frühere „unterentwickelte“ Gebiete) überfluten, zu reduzieren, ist es dennoch unwahrscheinlich, dass dies in absehbarer Zukunft die effektivste Therapie sein wird.

\section{Pharmakologische Hemmung der Thrombozyten- funktion: die drei „Klassiker“}

Bis in die 70er Jahre wurden die Thrombozyten hauptsächlich als ,unschuldige Zuschauer“ angesehen. Erst die langsam einsetzende Erkenntnis, dass die Behandlung mit Acetylsalicylsäure (Aspirin ${ }^{\circledR}$ ) die Rate der Herz-Kreislauf-ErkrankungendurchkovalenteThromboxanproduktionshemmung der Thrombozyten reduziert, löste eine Veränderung im Denken aus. Seitdem liegt das größte Problem darin, das offensichtliche Dilemma der Reduktion von Thrombosen zu meistern, ohne dabei die Hämostase zu beeinflussen, oder zumindest so wenig wie möglich! Denn bei beiden sind grundsätzlich die gleichen physiologischen Prozesse beteiligt. Die Frage, ob einige eine wichtigere Rolle bei der Thrombose als bei der Hämostase spielen, wird noch immer aktiv erforscht. Es gibt aber zumindest einige Anzeichen, die hier noch weitere Möglichkeiten eröffnen. Nach Acetylsalicylsäure war der nächste große Schritt vorwärts die Entdeckung der Thienopyridinfamilie (Ticlopidin und Clopidogrel), Thrombozyten- 
funktionsinhibitoren, die mittels kovalenter Blockade des ADP-Hauptrezeptors wirken [2]. Ticlopidin zeigte schwerwiegende Nebenwirkungen, weshalb heute Clopidogrel bevorzugt wird. Diese Präparate sind eigentlich „Vorstufen“, welche durch das Cytochrom P450 in der Leber aktiviert werden müssen, um dann mit dem P2 $\mathrm{Y}_{12}$-Rezeptor reagieren zu können. Bei normalen Personen sind etwa 70-80\% des $\mathrm{P}_{2} \mathrm{Y}_{12}$-Rezeptors inhibiert, was ungefähr den optimalen Wert für ein Gleichgewicht zwischen den Auswirkungen von Thrombose und Hämostase darstellt. Aus verschiedenen Gründen (vermutlich im Zusammenhang mit dem Leberstoffwechsel) lässt sich dieser optimale Wert u.U. nicht erreichen, und ein kleiner Teil der Thrombozyten muss somit als clopidogrelresistent betrachtet werden [3]. Eine Langzeitbehandlung mit Clopidogrel scheint jedoch einen Schutz gegen kardiovaskuläre Ereignisse zu bieten, stellt aber noch immer eine sehr kostspielige Alternative dar. Dies könnte sich mit dem Ablauf des Patentschutzes und der Möglichkeit der Herstellung von günstigeren Generika bald ändern.

Ein anderer Ansatz für die Thrombozytenfunktionshemmung bot die $\alpha_{\mathrm{II}} \beta_{3}$-Inhibition, die ursprünglich auf der Beobachtung der Glanzmann-Thrombasthenie basierte, bei welcher $\alpha_{\mathrm{IIb}} \beta_{3}$ fehlt oder fehlerhaft ist, sowie auch auf den Wirkungen von Schlangengift-Disintegrinen, die $\alpha_{\mathrm{IIb}} \beta_{3}$ blockieren. Die Aktivierung von $\alpha_{\mathrm{IIb}} \beta_{3}$ ist ein relativ spätes Ereignis auf dem gemeinsamen Weg der Thrombozyten, hervorgerufen durch sämtliche Agonisten. Der erste klinische Inhibitor war der humanisierte monoklonale Maus-Antikörper ReoPro ${ }^{\circledR}$ (Abciximab), der bei instabiler Angina pectoris und perkutaner Koronarintervention gut wirksam ist, indem er die Thrombozytenaggregation verhindert und daher die Thromboserate reduziert. Das zyklische Peptid Integrelin (Eptifibatid) oder das kleine Peptidomimetikum Aggrastat ${ }^{\circledR}$ (Tirofiban) wurden ebenso für diese Indikationen verwendet und sind gleichfalls wirksam [4]. Versuche, diese Methode zur Behandlung chronischer Krankheiten durch Entwicklung oraler Inhibitoren einzusetzen, führten jedoch zu Problemen. Obwohl man die Gründe dafür noch nicht ganz versteht, geht man davon aus, dass hauptsächlich eine inadäquate Thrombozytenhemmung, insbesondere Freisetzung von löslichem CD154 (sCD40L), und deshalb eine gesteigerte Thrombozyten-Endothelium- und Thrombozyten-Leukozyten-Interaktion dafür verantwortlich sind.

Daher besteht weiterhin ein großes Interesse an der Entwicklung neuer und wirkungsvollerer Thrombozy- tenhemmer zur Behandlung chronischer Krankheiten. Die Verhinderung des Thrombuswachstums sollte nicht das einzige Ziel sein; das Schicksal von teilweise aktivierten Thrombozyten, die nicht im Thrombus eingeschlossen sind, muss auch in Betracht gezogen werden. Man weiß wenig über ihre Regulierung, ob sie von der Zirkulation ausgeschlossen sind oder aber „,beruhigt“ werden durch Entfernung gefährlicher Rezeptoren wie P-Selectin und CD154, die während der Aktivierung exprimiert wurden. Es gibt auch Anzeichen dafür, dass sie Granula wieder aufladen können, um das hämostatische Potential zu erhalten oder zumindest große Blutungszwischenfälle abdecken zu helfen.

Der ADP-Rezeptor ist nach wie vor ein beliebter Forschungsgegenstand, und zwar deshalb, weil ADP, ungeachtet des ursprünglichen Agonisten, eine so wichtige Rolle bei der Thrombozytenaktivierung spielt. Zudem hat der P2Y $\mathrm{Y}_{12}$-Rezeptor den Vorteil, spezifisch von Thrombozyten und Nervenzellen exprimiert $\mathrm{zu}$ werden, und Letztere werden durch die Blut-Hirn-Schranke geschützt. Unter diesen Inhibitoren, die relativ weit entwickelt sind, findet man CS-747, ein anderes Thienopyridinmedikament [5]. Im Vergleich zu Clopidogrel hat es den Vorteil, dass geringere Dosen benötigt werden und es in der Leber rascher in die aktive Form umgewandelt wird. Andere Anti-P2 $Y_{12}$-Medikamente, die in oraler Darreichungsform erhältlich sind und nicht kovalent mit dem Rezeptor reagieren, befinden sich derzeit ebenfalls in der Entwicklungsphase. Experimentell gut erforschte Versionen wie AR-C69931MX [6] haben immer noch den Nachteil, dass sie Phosphatgruppen enthalten, welche in der Zirkulation weggespalten werden. Die neuere Forschung konzentriert sich auf Inhibitoren, die nicht von ADP oder ATP abgeleitet werden und keine leicht hydrolysierbaren Gruppen enthalten. Der Vorteil dieser nichtkovalenten Inhibitoren besteht darin, dass sie im Fall einer Blutung oder eines chirurgischen Notfalls schnell reversibel sind. Ihre Dosierung kann den Bedürfnissen des Patienten angepasst werden, ohne auf den Leberstoffwechsel Rücksicht zu nehmen. Gegenüber den $\alpha_{\mathrm{IIb}} \beta_{3}$-Inhibitoren haben die P2 $\mathrm{Y}_{12}$-Inhibitoren den Vorteil, ein breiteres „klinisches Fenster" anzubieten, so dass eine Unter- oder Überdosierung weniger wahrscheinlich ist.

Die Behandlung mit direkten Thrombininhibitoren wie Melagatran und seinem in oraler Darreichungsform erhältlichen Derivat Ximelagatran wirkt auch auf Thrombozyten - mittels Reduktion ihrer Aktivierung 
durch Spuren von Thrombin -, was Teil des Wirkmechanismus dieses Medikaments sein könnte [7].

Weiters bietet die Forschung Möglichkeiten für neue Ziele und Inhibitoren. Diese beinhalten GP Ib [8] und GP VI, Fraktalkine [9] und CD154 [10], gas6 und seine Rezeptoren [11]. Studien mit Knock-out-Mäusen, Antikörpern oder rekombinanten Proteinen haben gezeigt, dass sie alle eindeutige Vorteile zur Verhinderung von Thrombosen bieten, ohne dass man einen ,hämostatischen Preis“ dafür bezahlen müsste. Ohne ausgedehnte Studien mit Tieren wird es jedoch nicht möglich sein zu sagen, welche davon die besten Möglichkeiten bieten können. Zudem gibt es methodologische Probleme, wie z.B. die Tatsache, dass verschiedene tierische Thrombosemodelle ganz unterschiedliche Resultate zeitigen. So erbrachten Experimente mit laserverursachten Verletzungen nicht die gleichen Resultate wie jene mit $\mathrm{FeCl}_{3}$ hervorgerufene Beschädigung des Endotheliums [12], wenn die Auswirkung von rekombinantem GP VI versus Anti-GP-VI-Antikörper auf Thrombosen in Mäusen untersucht wurde [13]. Diese Beobachtung weist darauf hin, dass im Lasermodell Kollagen nicht exponiert wird und Thrombosen eher durch Aktivierung der Endothelzellen verursacht werden.

\section{Ein Ausblick in die Klinik}

Die kürzliche Aufregung um gehäufte Herzkrankheiten bei Patienten, die mit Cyclooxygenase-(COX-)2-Inhibitoren behandelt wurden, zeigt auch, wie vorsichtig man beim Verändern des Gleichgewichts zwischen Hemmung und Aktivierung im Gefäßsystem sein muss. Die COX-2-Inhibitoren [14] wirken u.a. auf das Endothelium der Gefäßwand ein und reduzieren die Prostacyclinproduktion. Dies impliziert, dass Gefäße die Tendenz haben, sich zu verengen, da die glatten Muskelzellen zu wenig entspannt sind. Gleichzeitig erhalten die Thrombozyten nicht die normale Menge von Prostacyclin, können aber weiterhin Thromboxan $\mathrm{A}_{2}$ produzieren und sind deshalb möglicherweise leichter aktivierbar. Bei Plaqueruptur könnte dieser Effekt zu einem größeren okkludierenden Thrombus führen.

Diabetes ist ebenfalls eine Indikation, bei der Thrombozytenhemmer öfter in Betracht gezogen werden sollten [15], da es zahlreiche Hinweise auf aktivere zirkulierende Thrombozyten gibt und viele Nebenwirkungen dieser Krankheit durch eine solche Behandlung zumindest reduziert werden könnten.

\section{Literatur}

1. Massberg S, Brand K, Gruner S, et al. A critical role of platelet adhesion in the initiation of atherosclerotic lesion formation. J Exp Med 2002; 196:887-96.

2. Herbert JM, Savi P. P2Y12, a new platelet ADP receptor, target of clopidogrel. Semin Vasc Med 2003;3:113-22.

3. Cattaneo M. Aspirin and clopidogrel: efficacy, safety, and the issue of drug resistance. Arterioscler Thromb Vasc Biol 2004;24:1980-7.

4. Silva MA, Gandhi PJ. Selection of glycoprotein Ilb/Illa inhibitors for upstream use in patients with diabetes experiencing unstable angina or non-ST segment elevation myocardial infarction. What have we learned in the last 10 years? J Clin Pharm Ther 2004;29:497-510.

5. Sugidachi A, Asai F, Ogawa T, et al. The in vivo pharmacological profile of CS-747, a novel antiplatelet agent with platelet ADP receptor antagonist properties. Br J Pharmacol 2000;129:1439-46.

6. Matsuno $\mathrm{H}$, Tokuda $\mathrm{H}$, Ishisaki A, et al. $\mathrm{P}_{2} \mathrm{Y}_{12}$ receptors play a significant role in the development of platelet microaggregation in patients with diabetes. J Clin Endocrinol Metab 2005;90:920-7.

7. Sarich TC, Osende JI, Eriksson UG, et al. Acute antithrombotic effects of ximelagatran, an oral direct thrombin inhibitor, and r-hirudin in a human ex vivo model of arterial thrombosis. J Thromb Haemost 2003;1:999-1004.

8. Cauwenberghs N, Meiring M, Vauterin S, et al. Antithrombotic effect of platelet glycoprotein Ib-blocking monoclonal antibody Fab fragments in nonhuman primates. Arterioscler Thromb Vasc Biol 2000; 20:1347-53.

9. Schafer A, Schulz C, Eigenthaler M, et al. Novel role of the membrane-bound chemokine fractalkine in platelet activation and adhesion. Blood 2004;103:407-12.

10. Andre P, Nannizzi-Alaimo L, Prasad SK, et al. Platelet-derived $\mathrm{CD}_{4} \mathrm{OL}$ : the switch-hitting player of cardiovascular disease. Circulation 2002; 106:896-9.

11. Angelillo-Scherrer A, Burnier L, Flores N, et al. Role of Gas 6 receptors in platelet signaling during thrombus stabilization and implications for antithrombotic therapy. J Clin Invest 2005;115:237-46.

12. Massberg S, Konrad I, Bultmann A, et al. Soluble glycoprotein VI dimer inhibits platelet adhesion and aggregation to the injured vessel wall in vivo. FASEB J 2004;18:397-9.

13. Gruner $S$, Prostredna $M$, Koch $M$, et al. Relative antithrombotic effect of soluble GPVI dimer compared with anti-GPVI antibodies in mice. Blood 2005;105:1492-9.

14. Frantz S. How to avoid another "Vioxx". Nat Rev Drug Discov 2005;4:5-7.

15. Ferroni P, Basili $S$, Falco A, et al. Platelet activation in type 2 diabetes mellitus. J Thromb Haemost 2004;2:1282-91.

\author{
Korrespondenzanschrift \\ Dr. Kenneth J. Clemetson \\ Theodor-Kocher-Institut \\ Universität Bern \\ Freiestraße 1 \\ 3012 Bern \\ Schweiz \\ Telefon (41/31) 6314148, Fax -9215443 \\ E-Mail:clemetson@tki.unibe.ch
}

